\title{
Towards Fully Automatic Placement of Security Sanitizers and Declassifiers
}

\section{Citation}

Livshits, Benjamin and Stephen Chong. 2012. Towards Fully Automatic Placement of Security Sanitizers and Declassifiers. Harvard Computer Science Group Technical Report TR-03-12.

\section{Permanent link}

http://nrs.harvard.edu/urn-3:HUL.InstRepos:29003506

\section{Terms of Use}

This article was downloaded from Harvard University's DASH repository, and is made available under the terms and conditions applicable to Other Posted Material, as set forth at http:// nrs.harvard.edu/urn-3:HUL.InstRepos:dash.current.terms-of-use\#LAA

\section{Share Your Story}

The Harvard community has made this article openly available.

Please share how this access benefits you. Submit a story. 


\title{
Towards Fully Automatic Placement of Security Sanitizers and Declassifiers
}

\author{
Benjamin Livshits \\ and \\ Stephen Chong
}

TR-03-12

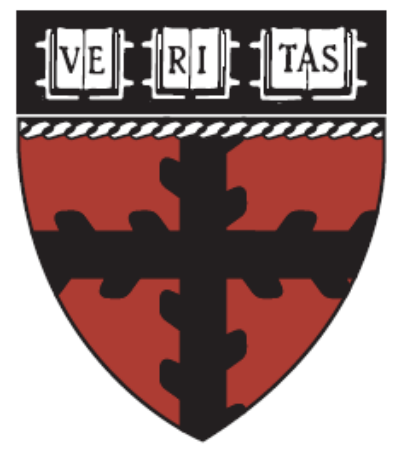

Computer Science Group

Harvard University

Cambridge, Massachusetts 


\section{Towards Fully Automatic Placement of Security Sanitizers and Declassifiers}

\author{
Benjamin Livshits \\ Microsoft Research
}

\author{
Stephen Chong \\ Harvard University
}

\begin{abstract}
A great deal of research on sanitizer placement, sanitizer correctness, checking path validity, and policy inference, has been done in the last five to ten years, involving type systems, static analysis and runtime monitoring and enforcement. However, in pretty much all work thus far, the burden on sanitizer placement has fallen on the developer. However, sanitizer placement in large-scale applications is difficult, and developers are likely to make errors, and thus create security vulnerabilities.

This paper advocates a radically different approach: we aim to fully automate the placement of sanitizers by analyzing the flow of tainted data in the program. We argue that developers are better off leaving out sanitizers entirely instead of trying to place them.

This paper proposes a fully automatic technique for sanitizer placement. Placement is static whenever possible, switching to run time when necessary. Run-time taint tracking techniques can be used to track the source of a value, and thus apply appropriate sanitization. However, due to the runtime overhead of run-time taint tracking, our technique avoids it wherever possible.
\end{abstract}

\section{Introduction}

Tracking of explicit information flow has received a great deal of attention in recent years. Two primary applications for explicit information flow tracking stand out prominently:

- preventing injection attacks within web applications such as cross-site scripting (XSS) and SQL injection; and

- preventing private data leaks, such as those recently observed in a variety of popular mobile applications [9].

These attacks have motivated a great deal of research in the last five to ten years on sanitizer placement, sanitizer correctness [11, 38], checking path validity, and policy inference $[19,34]$, involving type systems [28, 8], static analysis $[18,14,40,35,13,36]$, and run-time monitoring and enforcement $[10,7,6,21]^{1}$.

Much academic work in this space has focused on finding missing sanitizers and was performed on relatively small applications. Several projects have explored the use of run-time technique, motivated in part by the scalability and precision challenges that static analysis typically encounters. Additional motivation for exploring run-time techniques comes

\footnotetext{
${ }^{1}$ For simplicity, in the rest of this paper, we shall talk about sanitizer placement (for integrity preservation). Please note that our techniques apply equally well to the placement of declassifiers (for confidentiality preservation)
}

from the complexity of large-scale web applications with multiple, potentially nested sanitizers, which recent assessments [33, 32] suggest is well beyond the ability of developers to address using static reasoning and code reviews.

We also feel that the run-time approach is most practical in the long run, however, the overhead can be considerable. Prior work on sanitizer placement advocates dynamic sanitizer placement through a combination of inline instrumentation [21] and library-based instrumentation [7, 6]. The main advantage of library-based instrumentation is reduced overhead: only library code (as opposed to application code) needs to be instrumented. However, library-based approaches do not deal well with information propagated through non-library code such as char[], byte[], and custom character-level sanitizers. Custom character-level sanitizers are quite common, and sanitizers typically deal with string data at the level of characters [11]. The overhead of these approaches varies, but is generally between 1-20\%, depending on the application. In the case of library-based instrumentation, the "depth" of the data propagation path largely determines the overhead. In large enterprise applications, we know that data can undergo a high number of transformations during its lifetime [24], resulting in higher overhead than experiments with smaller applications would lead us to believe. We feel that it is crucial to develop novel ways to decrease the performance penalty for inline instrumentation to make it practical.

Prior research has proposed the use of pointer analysis as a way to reduce the number of instrumentation points [20, 2]. However, the number of objects that are deemed to be reachable from sources and may flow to sinks is still quite large in practice, leading to a high number of instrumentation points. Our approach proposes a secondary filter that, based on the structure of the interprocedural dataflow graph, obviates the need for instrumentation for many reachable nodes.

The approach this paper advocates is radically different: we aim to fully automate the placement of sanitizers by analyzing the flow of tainted data. A key observation is that, given a policy, sources and sinks within the application induce restrictions on the placement of sanitizers. It is difficult for developers to place sanitizers so as to satisfy all of these restrictions, especially in large-scale applications [33]. In fact, we argue that developers are better off leaving out sanitizers entirely, allowing them to be placed automatically.

In this paper we propose a fully automatic technique for sanitizer placement. The goal is to minimize both run-time overhead and code bloat due to instrumentation. Sanitizer placement is static whenever possible, switching to runtime techniques when necessary. We perform analyses on the inter-procedural dataflow graph of the program to identify 


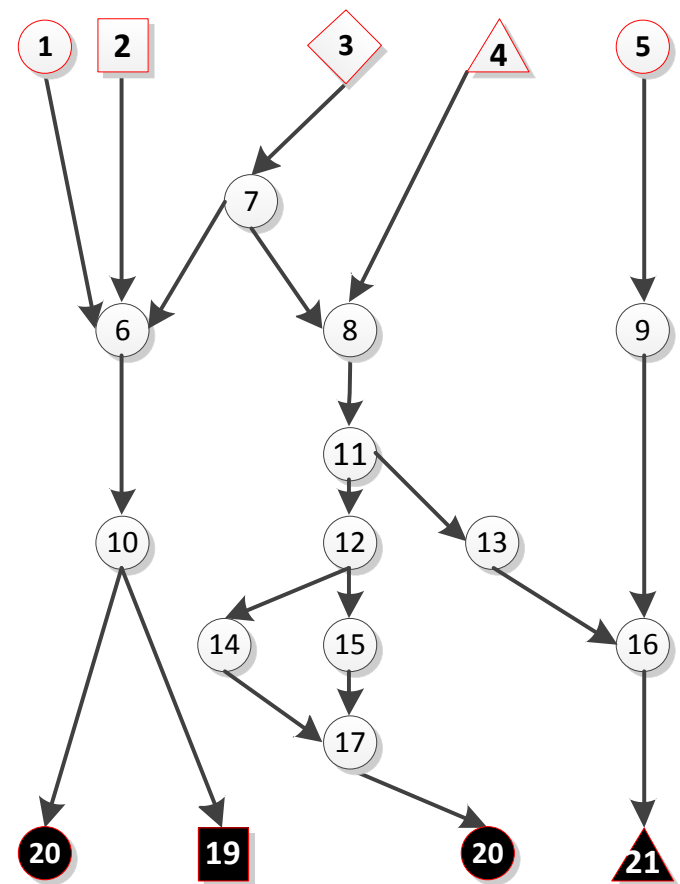

Figure 1. Motivating example of a small, but illustrative flow graph. Sources are at the top; sinks are at the bottom.

where sanitizers can be placed, and where values must be tracked at run-time in order to determine which sanitizer to apply to a value. In order to reduce run-time overhead, we resort to run-time tracking only when necessary.

\subsection{Sanitization Policies}

Large applications come with libraries of sanitizers. Developers are heavily discouraged from writing their own sanitizers. This is in part because most of the time, they get them wrong $[4,11]$. As a result, proper sanitizers are typically pure functions, whose signature is String $\rightarrow$ String.

Policies can be given in the form of a table, that for every type of data source and data sink provides the appropriate sanitizer to values that flow from that source to that sink. Policies are declarative specifications, and can both provide developer guidance and simplify the code review process. Section 2 gives examples of policies.

\subsection{Dataflow Graphs and Policies}

Figure 1 shows a dataflow graph for a simple program that will be used as an example throughout this paper. The policy for this example graph is shown in Figure 2. Source types $(\bigcirc, \square, \diamond$, $\triangle)$ are shown in rows and sink types $(\boldsymbol{O}, \mathbf{\square}, \mathbf{\Delta})$ are shown in columns. We use $\oslash$ as a special kind of source type and sink type, for data production

\begin{tabular}{|c|cccc|}
\hline & $\bullet$ & $\boldsymbol{口}$ & $\boldsymbol{\Delta}$ & $\oslash$ \\
\hline \hline$\bigcirc$ & $S_{1}$ & $S_{1}$ & $S_{4}$ & $\perp$ \\
$\square$ & $S_{1}$ & $S_{2}$ & $\perp$ & $\perp$ \\
$\diamond$ & $S_{2}$ & $S_{1}$ & $S_{3}$ & $\perp$ \\
$\triangle$ & $\perp$ & $\perp$ & $S_{3}$ & $\perp$ \\
$\oslash$ & $\perp$ & $\perp$ & $\perp$ & $\perp$ \\
\hline
\end{tabular}

Figure 2. Example policy. Sources shown vertically; sinks shown horizontally. $\perp$ means no sanitization required. or consumption that is not relevant to security (such as constant strings or other trusted sources of data). Thus, we assume that every source and sink of data has a type that appears in the table. Entries in the table indicate which sanitizer should be applied to data. We use metavariable $\mathcal{P}$ to range over policies, $I$ to range over source types, $O$ to range over sink types, and $S$ to range over sanitizers. We write $\mathcal{P}(I, O)$ for the entry in policy $\mathcal{P}$ for source $I$ and sink $O$. We assume that a node cannot be both a source and a sink, and write $\tau(n)$ for the source type or sink type of node $n$. For example, in Figure 1, where $n_{i}$ is the node labeled with integer $i$ we have $\tau\left(n_{3}\right)=\diamond$ and $\tau\left(n_{19}\right)=\boldsymbol{\square}$. Since $n_{11}$ is neither a source nor a sink, $\tau\left(n_{11}\right)$ is undefined.

For example, let $\mathcal{P}$ be the policy in Figure 2. Data originating from a source of type $\square$ and going to a sink of type should have sanitizer $S_{1}$ applied to it. If $\mathcal{P}(I, O)=\perp$ then no sanitization should be applied data flowing from source type $I$ to sink type $O$. This may indicate, for example, that constant string data should not be sanitized before being displayed to the user.

\subsection{Contributions}

This paper makes the following contributions:

- Fully Automatic sanitizer placement. We argue that sanitizer (and declassifier) placement should be done automatically, given a policy and an application, instead of the current approach of the developer being responsible for getting it right.

- Node-based placement. We propose a simple nodebased placement strategy for static sanitizer placement and show that, while simple to implement, it might not be adequate for complex dataflow graphs.

- Formalization of correctness. We introduce a theory of source- and sink-independent edges and formally define the validity of sanitizer placement.

- Edge-based placement. We propose an edge-based placement strategy for sanitizer placement, which attempts to place sanitizers statically and "spills over" into runtime whenever necessary.

- Experiments. We present a extensive evaluation of how our placement approach affects the number of instrumentation points on both large applications (up to 1.8 million lines of code) and synthetically generated dataflow graphs. While the node-based approach only instruments a fraction of all nodes (sometimes as little as $1-2 \%$ of tainted nodes), in most cases it fails to provide sanitization on all paths. The edge-based approach, while requiring more instrumentation points, provides full sanitization, while reducing the number of instrumentation points by as much as $2 \times$ or $4 \times$ in some cases. Our edgebased technique works even better in the case of a precise underlying dataflow analysis: for sparser synthetic graphs, the reduction in the number of instrumentation points is as high as $27 \times$.

\subsection{Paper Organization}

The rest of the paper is organized as follows. Section 2 presents examples that highlight the need for automated sanitizer placement. Section 3 gives an overview to our approach for automatic sanitizer placement. Section 4 presents dataflow analyses and algorithms to implement our approach. Section 5 describes our experimental evaluation. Finally, Sections 6 and 7 describe related work and conclude.

The companion technical report [17] contains more details. 


\section{Motivating Examples}

Our examination of large-scale applications has shown that more often than not, data processing is performed via a fixed set of sanitizers, whose proper selection depends on the kind of source and sink and can be captured via a table like the one shown in Figure 2.

Sanitization and declassification policies in this section illustrate the complexity of real-world data manipulation scenarios.

\subsection{Web Applications}

The OWASP Enterprise Security API (ESAPI) is an open-source web-

\begin{tabular}{|c|cc|}
\hline & URL & CSS \\
\hline \hline input & encodeForURL & encodeForCSS \\
\hline
\end{tabular}
application security library. The usage guidelines of the ESAPI reveal that the correct sanitization to apply to data depends on how the data will be used, that is, on the sink context. To sanitize a user-provided URL, the function ESAPI.encoder().encodeForURL(input) should be used. By contrast, to sanitize user input that will be used to construct a value for a CSS attribute, the function ESAPI.encoder (). encodeForCSS (input) should be used.

\subsection{Web Application Roles}

In large-scale web applications, sanitization requirements often vary based on who is interacting with the application. This is referred to as role-based sanitization. For example, Wordpress allows authors to insert certain HTML tags in their blog posts that commenters may not [39]. Similar approaches are taken by phpBB and Drupal. This complexity is reflected in sanitization libraries like Anti-XSS [23] or OWASP HTML Sanitizer Library [26], and HTML Purifier [41], where the developer can select different policies for sanitization of HTML.

This source-sensitivity arises because not all users are created equal, and that authentication provides a degree of trust (and increase of capabilities) that is not warranted for non-authenticated users.

\subsection{Encrypted Cloud}

Consider a web application using a public cloud provider for storage. The web application wants to use the cloud for scalability and to reduce storage hardware costs, but does not fully trust the cloud to protect the confidentiality of its data. The application therefore will use encryption when serializing data to the database, and decryption when deserializing.

In this scenario, the sources are of types input and cloud and sinks are of types outside/browser and cloud. The policy would encrypt data before it goes into the cloud and decrypt it on the way out of the cloud. The correct sanitization to apply (if any) depends on both the source and sink of data.

\subsection{Mobile App Privacy and Security}

Multiple studies have shown that applications on Android and other mobile platforms leak user data to untrusted parties. The basic idea is that the developer needs to filter out private data before it is allowed to go outside. However, the app often has legitimate reasons to send user input and data outside. Consider a gmail app that needs to communicate with its "parent" site, or its host, in this case, mail.google.com. It is necessary to send information to that hosting URL, including keystrokes, files on the local system if those are to be attached to email, etc. There is perhaps no compelling need to send user data to AdMob.com, a third-party mobile ad provider whose library is embedded in the app [9], and so data sent to a third-party should be cleansed, i.e., should have sensitive information removed. This highlights the need to treat the hosting site differently from third-party sites.

The source types for this scenario are user input, data from host, and data from third-party site. Sink types are screen output, isolated app storage, send to host, and send to third-party site. Data sent to a third party site that does not originate from the third party site should be cleansed. No other data cleansing is required, as shown below.

\begin{tabular}{|r|cccc|}
\hline & $\begin{array}{c}\text { screen } \\
\text { output }\end{array}$ & $\begin{array}{c}\text { isolated } \\
\text { app storage }\end{array}$ & $\begin{array}{c}\text { to } \\
\text { host }\end{array}$ & $\begin{array}{c}\text { to 3rd-party } \\
\text { site }\end{array}$ \\
\hline \hline user input & $\perp$ & $\perp$ & $\perp$ & cleanse \\
host & $\perp$ & $\perp$ & $\perp$ & cleanse \\
3rd-party site & ascii-sanitizer & $\perp$ & $\perp$ & $\perp$ \\
\hline
\end{tabular}

There are a few variations on this policy. For example, data that comes from a third-party and goes to the host might need to be either completely removed or cleansed to avoid untrusted and potentially maliciously crafted data reaching the host. Also, third-party data being shown to the screen might need to be pretty-printed or checked for integrity in some way, what in the table we refer to as asciisanitizer. Further, we assume that data from the host reaching a third-party site should be cleansed or removed entirely, because this data is user-specific.

\subsection{Properties of the Placement Problem}

Sink sensitivity: Sanitization is sink-sensitive: sanitization to apply to data depends on how the data will be used.

Source sensitivity: Sanitization is source-sensitive: the correct sanitizer to use on data depends on where the data comes from. Source sensitivity also makes full automatic sanitization (as advocated in Samuel et al. [32]) difficult.

Context-sensitivity: As elaborated in ScriptGard [33] and by [39], sanitization is also context-sensitive: to choose the proper sanitization strategy, the nested context needs to be determined. Consider the following snippet of HTML code, which displays a comment (the value untrusted) when the element is clicked.

<div class=' comment-box' onclick='displayComment (untrusted, this)'>

$</$ div $>$ ... hidden comment ...

The untrusted comment is in two nested contexts: it is in the onclick attribute of an HTML tag, and it is in a singlequoted JavaScript string context. To properly sanitize the untrusted comment, we must ensure that the untrusted comment does not contain either JavaScript or HTML metacharacters. In general, more than one sanitizer may be needed on a path between a given source and a sink. However, this can be still modeled by saying that for a given source and sink type, a single function representing the composition of sanitizers is required.

Not idempotent or reversible: Note that sanitizers are not guaranteed to be either idempotent or reversible, meaning that we cannot apply them more than once. A recent study [11] shows that out of 24 sanitizers considered, 19 are idempotent, and that only 2 are reversible. Moreover, order is important, as less than $30 \%$ of pairs of sanitizers commute. 


\section{Overview}

In this section we define the problem and present an overview of two solutions: a completely static node-based solution, and an edge-based solution that uses static analysis and run-time taint tracking.

\subsection{Valid Sanitizer Placement Problem}

We formulate our placement solution around the following correctness definition.

Definition 1. Given a dataflow graph $G=\langle N, E\rangle$, sanitization for the graph is valid for policy $\mathcal{P}$ if for all source nodes $s$, and all sink nodes $t$ :

- if $\mathcal{P}(\tau(s), \tau(t))=S$ then every value that flows from $s$ to $t$ has sanitizer $S$ applied exactly once, and no other sanitizer is applied.

- if $\mathcal{P}(\tau(s), \tau(t))=\perp$ then every value that flows from $s$ to $t$ has no sanitizer applied.

We require that a sanitizer be applied at most once on any given path because sanitizers are not necessarily idempotent [11]: applying it multiple times might result in incorrect computation. Also, we require that sanitizers are not applied needlessly. We also model multiple (potentially nested) sanitizers as a single (composite) sanitizer.

We consider two methods for finding a suitable placement of sanitizers in a program: a node-based formulation (Section 3.2) that is efficient, but may fail to produce a correct sanitization in all situations; and an edge-based formulation (Section 3.3) that always provides correct sanitization, but may require run-time taint tracking in order to determine the correct sanitizer to apply.

We assume that the dataflow graph $G=\langle N, E\rangle$ does not contain any node that has both multiple in-coming edges and multiple out-going edges. This assumption is without loss of generality, since if a graph does not satisfy this requirement, it can easily be transformed to one that does by the insertion of a synthetic node. This assumption is required for the correctness of the edge-based formulation, and is analogous to assumptions in control-flow graph analysis of the absence of critical edges: edges that go from a node with multiple successors to a node with multiple predecessors.

\subsection{Node-based Formulation}

We say that a node $n$ is $S_{i}$-possible if it is on a path from a source node $s$ to a sink node $t$ that requires sanitizer $S_{i}$, that is, $\mathcal{P}(\tau(s), \tau(t))=S_{i}$. Thus, if $n$ is $S_{i}$-possible, then at least some of the data passing through node $n$ requires application of $S_{i}$. We say a node $n$ is $S_{i}$-exclusive if all data passing $n$ requires application of $S_{i}$. In other words, node $n$ is $S_{i}$-exclusive if it is $S_{i}$-possible, and for all $j \neq i$, it is not $S_{j}$-possible.

Definition 2. Node $n \in N$ is $S_{i}$-possible if there is a source node $s$ and sink node $t$ such that $n$ is on a path from $s$ to $t$ and $\mathcal{P}(\tau(s), \tau(t))=S_{i}$.

Definition 3. Node $n \in N$ is $S_{i}$-exclusive if it is $S_{i}$-possible and for all source nodes $s$ and sink nodes $t$, if $n$ is on a path from $s$ to $t$ then $\mathcal{P}(\tau(s), \tau(t))=S_{i}$.

Figure 3 shows possible and exclusive nodes for sanitizers $S_{1}, S_{2}, S_{3}$, and $\perp$. Note that while possible nodes are plentiful, exclusive nodes are much more difficult to come by. In fact, $S_{2}$ and $\perp$ have no exclusive nodes associated with them at all. Note that node 13 is on a path both from node 3 to 21 , and from node 4 to 21 . However, it are $S_{3}$-exclusive because both $\tau(3)=\diamond$ and $\tau(4)=\triangle$ require the same sanitizer when going to $\operatorname{sink} \mathbf{\Delta}: \mathcal{P}(\diamond, \mathbf{\Delta})=\mathcal{P}(\triangle, \mathbf{\Delta})=S_{3}$.

For sparser

dataflow graphs,

exclusive nodes will

be more plentiful.

Exclusive nodes are

good candidates at

which to apply a

sanitizer to all data

passing through

the node. However,

exclusive nodes are

\begin{tabular}{llr}
\hline \multicolumn{1}{c}{ Possible } & Exclusive \\
\hline$S_{1}$ & $1,2,6,10,18,19$ & 1 \\
$S_{2}$ & $2,3,6,7,8,10,11,12$ & \\
& $14,15,17,20$ & \\
$S_{3}$ & $3,4,7,8,11,13,16,21$ & 13 \\
$S_{4}$ & $5,9,16,21$ & 5,9 \\
$\perp$ & $4,8,11,12,14,15,17,20$ & \\
\hline
\end{tabular}

Figure 3. $S_{i}$-possible and $S_{i^{-}}$ exclusive nodes for Figure 1.

not necessarily unique: there may be multiple $S_{i}$-exclusive nodes on a single path from a source to a sink. If there are multiple $S_{i}$-exclusive nodes on a path, we need to choose just one of them at which to apply sanitizer $S_{i}$. Since in many common applications of data sanitization, a sanitized value is larger than the unsanitized value (e.g., escaping special characters in a string will increase the length of the string), we prefer to perform sanitization as late as possible. We say that node $n$ is $S_{i}$-latest-exclusive if it is $S_{i}$-exclusive, and for every path going through $n$, it is the last $S_{i}$-exclusive node on that path.

Definition 4. Node $n \in N$ is $S_{i}$-latest-exclusive if $n$ is $S_{i}$ exclusive and for every source node $s$ and sink node $t$, and for every path from $s$ to $t$, if $n$ is on that path, then $n$ is the last $S_{i}$ exclusive node on the path.

By this definition, we see that in Figure 1 nodes 1, 9, and 13 are latest exclusive nodes (for sanitizers $S_{1}, S_{4}$ and $S_{3}$ respectively). Node 5 is not an $S_{4}$-latest exclusive node, since there is another $S_{4}$ exclusive node later on a path from node 5 . Indeed, it is easy to see from the definition that for any path from source node $s$ to sink node $t$ with $\mathcal{P}(\tau(s), \tau(t))=S_{i}$, there is at most one $S_{i}$-latest-exclusive node on that path. There may, however, be no $S_{i}$-latestexclusive node on a path, meaning that the node-based placement may fail to correctly sanitize all values.

\subsection{Edge-based Formulation}

We consider instead an edge-based formulation that is able to always find a correct placement of sanitizers in a dataflow graph, although it may be necessary to record and track at run time some information about the path that a value has taken in the graph in order to determine the correct sanitizer (if any) to apply to the value.

Figure 4 summarizes the key concepts used in our edgebased solution. We provide full definitions and intuition for each of these terms below.

We say that an edge $e$ is source-dependent if the sanitization to apply to values traversing $e$ depends on which source produced the value. Similarly, we say an edge is sinkdependent if the sanitization to apply to values traversing it depends on which sink the value will go to.

Definition 5. An edge e is source-dependent if there exists sources $s_{0}$ and $s_{1}$ and sinks $t_{0}$ and $t_{1}$ such that $e$ is on a path from $s_{0}$ to $t_{0}$ and on a path from $t_{1}$ to $t_{1}$ and either $\mathcal{P}\left(\tau\left(s_{0}\right), \tau\left(t_{0}\right)\right) \neq \mathcal{P}\left(\tau\left(s_{1}\right), \tau\left(t_{0}\right)\right)$ or $\mathcal{P}\left(\tau\left(s_{0}\right), \tau\left(t_{1}\right)\right) \neq$ $\mathcal{P}\left(\tau\left(s_{1}\right), \tau\left(t_{1}\right)\right)$. (That is, for at least one of sink $t_{0}$ or $t_{1}$, the sanitizer to use depends on the source.)

Definition 6. An edge $e$ is sink-dependent if there exists sources $s_{0}$ and $s_{1}$ and sinks $t_{0}$ and $t_{1}$ such that $e$ is on a path from $s_{0}$ to $t_{0}$ and on a path from $s_{1}$ to $t_{1}$ and 


\begin{tabular}{ll}
\hline \multicolumn{1}{c}{ Term } & \multicolumn{1}{c}{ Brief description } \\
\hline Source-dependent edge & Sanitizer to apply to values traversing the edge depends on which source type the value came from. \\
Sink-dependent edge & Sanitizer to apply to values traversing the edge depends on which sink type the value will go to. \\
In-trigger edge & Source-independent edge with a source-dependent successor. \\
Out-trigger edge & Source-independent edge with a source-dependent predecessor. \\
Sanitization edge & Earliest sink-independent edge on some path from a source to a sink. \\
Tag edge & In-trigger edge that isn't dominated by sanitization edges. Start runtime tagging of values. \\
Untag edge & Either a sanitization edge, or an out-trigger edge that isn't dominated by sanitization edges. Stop runtime \\
& tagging of values. \\
Carry edge & Edge that (a) is reachable from a tag edge without an intervening untag edge, and (b) can reach an \\
& untag edge, and (c) is neither a tag nor an untag edge. Instrument to propagate runtime taint values. \\
\hline
\end{tabular}

Figure 4. Summary of terms for edge-based placement.

either $\mathcal{P}\left(\tau\left(s_{0}\right), \tau\left(t_{0}\right)\right) \neq \mathcal{P}\left(\tau\left(s_{0}\right), \tau\left(t_{1}\right)\right)$ or $\mathcal{P}\left(\tau\left(s_{1}\right), \tau\left(t_{0}\right)\right) \neq$ $\mathcal{P}\left(\tau\left(s_{1}\right), \tau\left(t_{1}\right)\right)$. (That is, for at least one of source $s_{0}$ or $s_{1}$, the sanitizer to use depends on the sink.)

Intuitively, if an edge is sink-dependent, then when a value traverses the edge, we do not yet know which sanitizer to apply. By contrast, if an edge is source-dependent, we do not know which sanitizer to apply to values traversing the edge unless we know from which source the value originated. If an edge is neither source-dependent nor sink-dependent, then all values traversing the edge are meant to have the same sanitizer applied.

We say that edge $e$ is source (sink) independent if it is not source (sink) dependent.

In Figure 1, the edge from node $n_{6}$ to node $n_{10}$ is both source dependent and sink dependent. It is sink dependent because it is on a path from $n_{1}$ to both $n_{18}$ and $n_{19}$, but $\mathcal{P}\left(\tau\left(n_{1}\right), \tau\left(n_{18}\right)\right)=S_{1} \neq S_{2}=\mathcal{P}\left(\tau\left(n_{1}\right), \tau\left(n_{19}\right)\right)$. It is source dependent since it is on a path from both $n_{1}$ and $n_{2}$ to $n_{19}$ and $\mathcal{P}\left(\tau\left(n_{1}\right), \tau\left(n_{19}\right)\right)=S_{1} \neq S_{2}=\mathcal{P}\left(\tau\left(n_{2}\right), \tau\left(n_{19}\right)\right)$.

The edge from node $n_{7} n_{8}$ is source independent (since only one source node can reach it), but is sink dependent.

\subsubsection{Trigger Edges}

To apply a sanitizer at a source-dependent edge, we must know from which source a value originated. We can use runtime tracking to "taint" a value so that we can determine its source. However, run-time taint tracking can be expensive, and we do not need to track all values manipulated by the system, just those for which we need to know the source in order to determine which sanitizer to apply.

We identify edges where it is necessary to start run-time tracking of values, and edges where, if we were tracking, if suffices to stop tracking. Edge $e$ is an in-trigger edge if it is a source-independent edge but has an edge after it that is source-dependent. In-trigger edges are the edges where we have sufficient information to know where a value came from, and need to start run-time tracking because the origin of a value affects which sanitizer to apply.

Definition 7. Edge e is an in-trigger edge if it is a sourceindependent edge from node $n_{1}$ to node $n_{2}$ such that there exists a source-dependent edge $n_{2} \rightarrow n_{0}$.

Edge $e$ is an out-trigger edge if it is a source independent edge that is preceded by a source-dependent edge $e^{\prime}$. If we were tracking run-time values as they traverse edge $e^{\prime}$, then we no longer need to track them when they traverse edge $e$.

Definition 8. Edge $e$ is an out-trigger edge if it is a sourceindependent edge from node $n_{1}$ to node $n_{2}$ such that there exists a source-dependent edge $n_{0} \rightarrow n_{1}$.

Once we have sanitized a value, we will not need to perform run-time tracking for the value. (This is an invariant that our run-time discipline will enforce: only values that require sanitization and have not yet been sanitized will be tagged at run time.) Because run-time tracking of values can be expensive, we typically want to perform sanitization as early as possible. We can only perform sanitization at sinkindependent edges (because at sink-dependent edges, the sanitization to apply depends on the future use of the value). Sanitization edges are the earliest possible edges at which we can perform sanitization: they are sink-independent edges that are the earliest sink-independent edge for some path from a source to a sink. That is, if $e$ is a sanitization edge, then for at least one path from a source to a sink, it is the earliest sink-independent edge.

Definition 9. Edge $e$ is a sanitization edge if it is a sinkindependent edge and there is a source node $s$ and sink node $t$ such that $e$ is the earliest sink-independent edge on a path from $s$ to $t$.

Figure 5 shows the source and sink dependent edges, in-trigger edges, out-trigger edges, and sanitization edges for our running example from Figure 1. For example, edge $n_{4} \rightarrow n_{8}$ is an in-trigger edge, since it is source independent, but has a successor edge $n_{8} \rightarrow n_{11}$ that is source dependent. Edge $n_{10} \rightarrow n_{19}$ is a sanitization edge as it is the earliest sink-independent edge on the path from not $n_{2}$ to $n_{19}$. Note that edge $n_{13} \rightarrow n_{16}$ is not a sanitization edge, even though it is sink independent. This is because any path that goes through $n_{13} \rightarrow n_{16}$ must first go through the sink independent edge $n_{11} \rightarrow n_{13}$.

In addition, for each edge $e$ in the graph, Figure 5 shows the policy table at $e$. This is simply the policy table $\mathcal{P}$ restricted to the source types $I$ and sink types $O$ such that $e$ is on a path from a source node of type $I$ to a sink node of type $O$. Policy tables at edges are a useful concept for computing an appropriate placement, and will be used in Section 4 .

Definition 10. The edge policy at edge $e$ is the restriction of the (global) policy $\mathcal{P}$ to only include source types $I$ and sink types $O$ such that $e$ is on a path from a source node of type $I$ to a sink node of type $O$. We write $\mathcal{P}_{e}$ for the edge policy at edge $e$.

\subsubsection{Tag, Untag, and Carry edges}

In-trigger edges and out-trigger edges help us identify where we may need to start, and can stop, run-time tracking of values. However, we can refine these notions to reduce the amount of run-time tracking we must perform.

Intuitively, run-time tracking is necessary only when a sanitization edge needs to distinguish values coming from different sources. These are exactly the sanitization edges that are source dependent. We need to propagate taint 
information only along source-dependent edges, and only until we sanitize the value.

This also means that we only need to start taint tracking (which we refer to as "tagging" data) when data values move from a source-independent edge to a source-dependent edge and the data is not yet sanitized (and will need sanitization in the future). Similarly, we can stop taint tracking (which we refer to as "untagging" data) when tagged data is sanitized, or when it moves from a source-dependent edge to a sourceindependent edge.

Specifically, a tag edge (where we tag values at run time, and start the run-time traint tracking) are in-trigger edges such that a value traversing the edge might be unsanitized and require sanitization in the future. A value is unsanitized if it has not gone through a sanitization edge, and thus the tag edges are in-trigger edges that are not dominated by a sanitization edge. Note that we assume that an edge dominates itself, and thus a tag edge cannot also be a sanitization edge.

Definition 11. Edge $e$ is a tag edge if $e$ is an in-trigger edge that is not dominated by sanitization edges.

An untag edge is an edge such that a tagged value can reach it (i.e., it is not dominated by sanitization edges), and we no longer need to track the tagged values. It is either a sanitization edge (since after sanitization we no longer need to track taint), or an out-trigger edge.

Definition 12. An untag edge is either

- an out-trigger edge that is not dominated by a sanitization edge or

- a sanitization edge.

At tag edges we tag values and start taint tracking, and continue taint tracking the value until the value reaches an untag edge: if the untag edge is an out-trigger edge then we can stop taint tracking; if the untag edge is a sanitization edge, we apply the appropriate sanitizer. (Note that if we stop taint-tracking a value at an untag edge, we may potentially resume taint tracking if the value later encounter another in-trigger edge not dominated by sanitization edges.) Edges between tag edges and untag edges will need to propagate tag values. We refer to these edges as carry edges.

Definition 13. Edge $e$ is a carry edge if $e$ is on a path from a tag edge to an untag edge such that the path does not contain an untag edge. That is, if edges $e_{0}, \ldots, e_{n}$ are a path where $e_{0}$ is a tag edge, $e_{n}$ is an untag edge, and $e_{1}, \ldots, e_{n-1}$ are not tag edges, then edges $e_{1}, \ldots, e_{n-1}$ are carry edges.

In Figure 5, edge $n_{7} \rightarrow n_{6}$ is a tag edge: it is an intrigger edge (since it is source independent and successor edge $n_{6} \rightarrow n_{10}$ is source dependent) that is not dominated by sanitizer edges. By contrast, edge $n_{13} \rightarrow n_{16}$ is an intrigger edge, but it is not a tag edge, since it is dominated by sanitizer edge $n_{11} \rightarrow n_{13}$. This means that any values traversing $n_{13} \rightarrow n_{16}$ will already be sanitized, and so there is no need to track their source type in order to determine which sanitizer to apply. In Figure 5, all untag edges are sanitization edges.

Edge $n_{6} \rightarrow n_{10}$ is a carry edge, as it is on a path from tag edge $n_{1} \rightarrow n_{6}$ to untag edge $n_{10} \rightarrow n_{18}$ without an intervening untag edge. Edge $n_{6} \rightarrow n_{10}$ will propagate the tags that tag edges $n_{1} \rightarrow n_{6}, n_{2} \rightarrow n_{6}$, and $n_{7} \rightarrow n_{6}$ create, enable sanitization edges $n_{10} \rightarrow n_{18}$ and $n_{10} \rightarrow n_{18}$ to apply the appropriate sanitization.

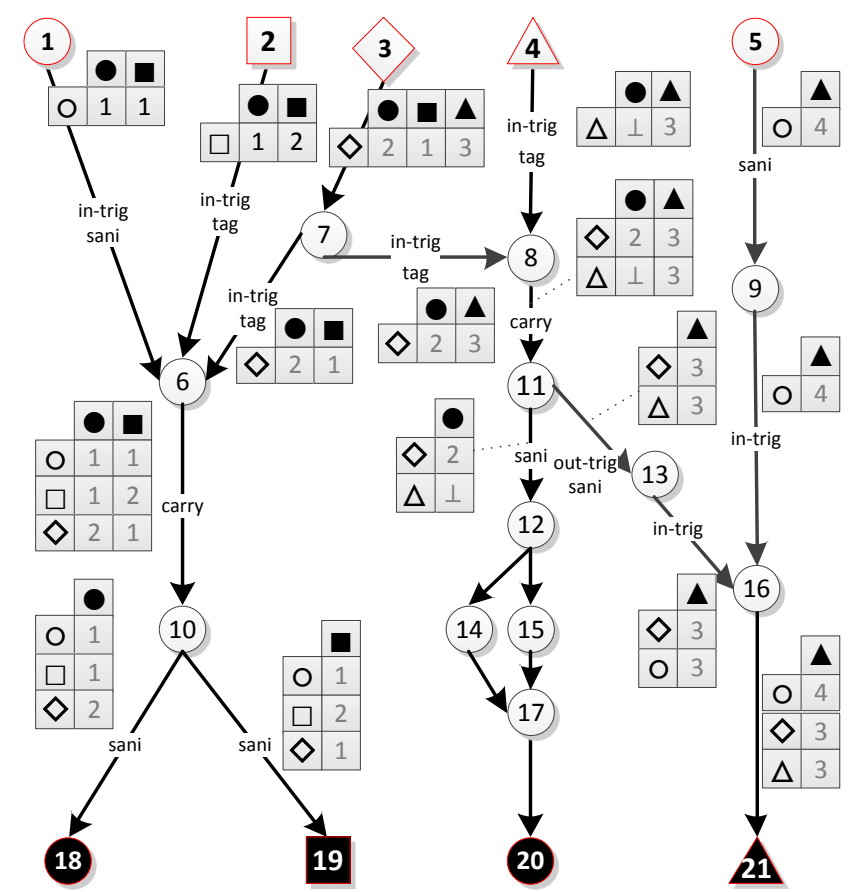

Figure 5. Policy tables are shown at every node and trigger edges are marked.

\subsubsection{Run-Time Taint Tracking for Sanitization}

We have defined several different kinds of edges that are relevant to the run-time discipline for applying correct sanitization to values: sanitization edges, tag edges, untag edges, and carry edges. We summarize what the instrumentation for these edges is required to do at run time:

- tag edge: when a value passes through a tag edge, if the value is not tagged then tag the value with one of the source types reaching it. A reaching source node, and its type, can be statically determined by examining the edge policy.

- untag edge: when a tagged value passes through an untag edge, untag it.

- sanitization edge: If the sanitization edge is not preceded by a carry edge, then no tagged values can reach this edge, and all values traversing this edge should have the same sanitizer applied. Otherwise, apply sanitization only if the value is tagged by looking up the tag (which is a source type) in the edge's policy table to find the appropriate sanitizer to apply (which might be $\perp$, in which case no sanitization is applied).

- carry edge: when a value passes through a carry edge, any taint on the value must be propagated.

For example, in Figure 5, consider a value flowing from source node $n_{3}$ to sink node $n_{20}$. At tag edge $n_{7} \rightarrow n_{8}$, the value will be tagged with it's originating source type $\tau\left(n_{3}\right)=\diamond$. The value with it's tag will be propagated over carry edge $n_{8} \rightarrow n_{11}$. Upon reaching sanitization edge $n_{11} \rightarrow n_{12}$, its tag will be examined, and the appropriate sanitizer $\left(S_{3}\right)$ applied. Note that the tag was needed for $n_{11} \rightarrow n_{12}$ to determine which sanitizer to apply, since value from source node $n_{4}$ could also traverse that edge, requiring no santization $(\perp)$. 


\subsubsection{Correctness of Edge-based Placement}

The edge-based placement produces a valid placement (Definition 1). We present here the key lemma that proves this.

Lemma 1. For every path from a source node $s$ to a sink node $t$, the following conditions hold for values flowing along that path.

1. There is at least one sanitization edge on the path.

2. If $\mathcal{P}(\tau(s), \tau(t))=\perp$ then no sanitization will be applied.

3. If $\mathcal{P}(\tau(s), \tau(t)) \neq \perp$ then sanitizer $\mathcal{P}(\tau(s), \tau(t))$ will be applied at the first sanitization edge.

4. No sanitization will be applied at the second or subsequent sanitization edges.

Proof: Condition (1) holds from the definition of sanitization edges, and because an edge whose target is a sink node is sink-independent.

Let $e_{0}, \ldots, e_{n}$ be a path from a source node to a sink node, and let $e_{i}$ be the first sanitization edge. Conditions (2) and (3) hold by the following argument. If $e_{i}$ is source independent then all values passing $e_{i}$ will have the same sanitization applied (either sanitizer $S$ if $\mathcal{P}(\tau(s), \tau(t))=S$, or no sanitization if $\mathcal{P}(\tau(s), \tau(t))=\perp)$. Suppose that $e_{i}$ is source dependent. Then there must be some other source $s^{\prime}$ such that there is a path from $s^{\prime}$ to $e_{i}$ and some output $t^{\prime}$ reachable from $e_{i}$ such that $\mathcal{P}\left(\tau(s), \tau\left(t^{\prime}\right)\right) \neq \mathcal{P}\left(\tau\left(s^{\prime}\right), \tau\left(t^{\prime}\right)\right)$. Since $e_{0}$ is source independent and $e_{i}$ is source dependent and the first sanitizer edge, there must be some edge $e_{j}$ on the path $e_{0}, \ldots e_{i-1}$ such that $e_{j}$ is a tag edge, and all edges $e_{j+1}, \ldots, e_{i-1}$ are carry edges. Thus, at $e_{j}$, the value will be tagged with source type $\tau(s)$ (or some other source type $I$ such that $\mathcal{P}(\tau(s), \tau(t))=\mathcal{P}(I, \tau(t)))$, the carry edges will propagate this tag, and so at sanitization edge $e_{i}$, the correct sanitization will be applied.

Suppose that condition (4) doesn't hold. Then there is some edge $e_{k}$ in path $e_{i+1}, \ldots, e_{n}$ such that $e_{k}$ is a sanitization edge, and $e_{k}$ applies sanitization to values traversing path $e_{o}, \ldots, e_{n}$. Since $e_{k}$ is a sanitizatio edge, it is the earliest sink-independent edge on some path from source to a sink, and so there must be some other source node $s^{\prime}$ that can reach $e_{k}$. Since $e_{k}$ is the first sink-independent edge on a path from $s^{\prime}$, there must be another edge leaving source $\left(e_{k}\right)$ (where $\operatorname{source}(e)$ denotes the source node of edge $e$ ) such that on that edge, some sink node $t^{\prime}$ is reachable that is not reachable from $e_{k}$, and $\mathcal{P}\left(\tau\left(s^{\prime}\right), \tau(t)\right) \neq \mathcal{P}\left(\tau\left(s^{\prime}\right), \tau\left(t^{\prime}\right)\right)$. More over, since source $\left(e_{k}\right)$ has multiple edges coming from it, by assumption that the dataflow graph has no nodes with both multiple successors and multiple predecessors, node source $\left(e_{k}\right)$ has a single predecessor, edge $e_{k-1}$, and so $e_{k-1}$ is also on the path from $s^{\prime}$ to $e_{k}$. Therefore, edge $e_{k-1}$ must be source dependent: since $e_{i}$ can reach $t^{\prime}$, and $e_{i}$ is sink-independent, it means that either $\mathcal{P}\left(\tau(s), \tau\left(t^{\prime}\right)\right) \neq$ $\mathcal{P}\left(\tau\left(s^{\prime}\right), \tau\left(t^{\prime}\right)\right)$ or $\mathcal{P}(\tau(s), \tau(t)) \neq \mathcal{P}\left(\tau\left(s^{\prime}\right), \tau(t)\right)$.

Now consider whether $e_{k-1}$ is a carry edge.

- Suppose $e_{k-1}$ is a carry edge.. We will show that none of the edges on $e_{i}, \ldots, e_{k-1}$ can be a tag edge, and thus, a value coming from $e_{i}$ cannot be tagged, and so at sanitizer $e_{k}$, no sanitization will be applied. This contradicts the assumption that condition (4) doesn't hold.

Note that $e_{i}$ is not a tag edge, as it is a sanitization edge. Then there must be some tag edge $e_{m}$ between $e_{i}$ and $e_{k-1}$. Since it is not dominated by sanitizer edges, there must be a path from a source node $s_{0}$ (such that $\left.\tau\left(s_{0}\right) \neq \tau(s)\right)$ to $\operatorname{source}\left(e_{m}\right)$ without a sanitization edge.
Since $e_{m}$ is an in-trigger edge, it is source independent. That means that for all sink types $O$ reachable from $e_{m}$, and all source types $I$ that can reach $e_{m}$, we have $\mathcal{P}_{e_{m}}(\tau(s), O)=\mathcal{P}_{e_{m}}(I, O)$. But any sink type $O$ reachable from $e_{m}$ is also reachable from $e_{i}$, and $e_{i}$ is sink independent. That means that for any sink types $O_{1}$ and $O_{2}$ we have $\mathcal{P}_{e_{m}}\left(\tau(s), O_{1}\right)=\mathcal{P}_{e_{m}}\left(\tau(s), O_{2}\right)$. Together these imply that $e_{m}$ is sink-independent, since for any source $I$ that can reach $e_{m}$ and sinks $O_{1}$ and $O_{2}$ that can be reached from $e_{m}$ we have:

$$
\begin{array}{rlr}
\mathcal{P}_{e_{m}}\left(I, O_{1}\right) & =\mathcal{P}_{e_{m}}\left(\tau(s), O_{1}\right) & e_{m} \text { is source independent } \\
& =\mathcal{P}_{e_{i}}\left(\tau(s), O_{1}\right) & O_{1} \text { is also reachable from } e_{i} \\
& =\mathcal{P}_{e_{i}}\left(\tau(s), O_{2}\right) & e_{i} \text { is sink independent, } \\
& =\mathcal{P}_{e_{m}}\left(\tau(s), O_{2}\right) \\
& =\mathcal{P}_{e_{m}}\left(I, O_{2}\right) &
\end{array}
$$

But then $e_{m}$ is the first sink-independent edge on the path from $I_{0}$ to $e_{m}$, and so it is a sanitization edge. This is a contradiction, as $e_{m}$ is a tag edge.

- Suppose $e_{k-1}$ is not a carry edge. Edge $e_{k}$ is an untag edge (since it is a sanitization edge). Note that $e_{k-1}$ is not a tag edge, since it is source dependent. But since $e_{k-1}$ is source dependent, and $e_{k}$ is the first sanitization edge on the path from $s^{\prime}$ to $e_{k}$, then there must be a tag edge on the path from $s^{\prime}$ to $e_{k}$ without any intervening untag edges between it and $e_{k}$. Therefore $e_{k-1}$ is a carry edge, which is a contradiction.

The correctness of the edge-based placement follows trivially from Lemma 1 and the fact that no edge other than a sanitizer edge applies sanitization.

\subsubsection{Optimizations}

There are several opportunities for optimization in the edgebased placement approach described above.

Remove un-needed sanitization edges: For simplicity of the presentation and the proof, we have defined the behavior of tag edges and sanitization edges treating "no sanitization" $\perp$ as if it were a sanitizer. If a sanitizer is not preceded by a carry edge, and the policy dictates that no sanitization should be applied, then the sanitization edge does not perform any computation, and should not be instrumented. Similarly, if a tag edge is tagging a value with a source type that will never require sanitization, then the tag edge can be removed, and the value never tagged. This optimization is valid because a sanitization edge that may receive tagged values will never sanitize an untagged value.

Sanitization edges preceeded by carry edges: For simplicity we required that any sanitization edge preceeded by a carry edge needed to check the runtime tag before applying sanitization. There are some situations (statically determinable) where a sanitization edge will be preceeded by a carry edge, yet all values going through it should have the same sanitization applied. In Figure 5 edge $n_{11} \rightarrow n_{13}$ is an example of this: the preceeding edge $n_{8} \rightarrow n_{11}$ is a carry edge, but $n_{11} \rightarrow n_{13}$ is source independent, and (importantly) is the first sanitization edge on any path that goes through it. Thus, all values traversing $n_{11} \rightarrow n_{13}$ will have sanitizer $S_{3}$ applied, and there is no need to examine the tag. 
Attaching tags to run-time values: We envision the runtime taint tracking being implemented simply by attaching tags to run-time values. This is a strategy that works well for dynamic languages such as Java, PHP, or JavaScript. The tags can be quite compact: we have described it above as tagging a value with the source that it originated from (or a source that has equivalent sanitization requirements), but it would suffice use bit strings that uniquely identify a source type. The number of sources depends on the policy, but will typically be small, meaning that a tag of 3-4 bit would suffice. There are opportunities for efficient implementation of taint-tracking when the tags are this small, such as placing the tag within the value header at run time.

With the tagging approach, the instrumentation for carry edges becomes trivial, since tags will be copied if they exist. Thus, the only instrumentation required will be to tag values as they pass through tag edges, untag them as they pass through untag edges, and apply appropriate sanitization at sanitization edges.

Efficient lookup for sanitization: Since the number of possible tags that can reach a given sanitization edge is small and known statically, we can pre-compute a lookup table for each sanitization edge that maps the tag number to the required sanitizer, thus minimizing run-time calculations.

\section{Placement Algorithms}

In this section, we propose concrete algorithms for computing the sets and relations described in Section 3. At the core of these computations, we have dataflow analysis, as described in Aho et al. [1]. As we will see, we can often stage our computation and break it town into a series of two or three analyses, one after another. As Knoop et al. [15] observe, this is often advantageous compared to a more complex equation-based approach, because each analysis stage completes fast.

\subsection{Node-based Placement}

Section 3.2 suggests a node-based placement approach. We will proceed to compute the set of nodes that are $S_{i}$-possible and $S_{i}$-exclusive, with respect to each sanitizer $S_{i}$ for $i$ ranging from 1 to $k$. To combine the computation of these properties for different sanitizers $S_{i}$, we use bitvectors as our representation. Generally, a 1 at position $i$ for a value at node $n \in N$ means that the property (either possiblity or exclusiveness) holds for $S_{i}$.

First, we compute available source types and anticipated sink types at every node using a dataflow analysis, as shown in Figure 6. We specify dataflow analyses by giving the semilattice of dataflow facts, the initial values of source nodes, the transfer function for nodes, and the direction of the dataflow analysis. This is a complete specification of the dataflow analyses.

We then combine the available sources and anticipated sinks information as described in Algorithm 1, to determine for each node which sanitizers are possible at every node. This is done by projecting the policy table to only the available source types and anticipated sink types. We write $\operatorname{Project}(\mathcal{P}, S, T)$ for the policy table that contains only the rows of policy table $\mathcal{P}$ for sink types $S$, and only the columns of $\mathcal{P}$ for sink types $T$. If at node $n$, sanitizer $S_{i}$ appears in the projected policy table $\operatorname{Project}(\mathcal{P}$, available $(n)$, anticipated $(n))$, then $n$ is on a path from a source to a sink that requires $n$ is $S_{i}$-possible.

Algorithm 1. Possible nodes.

\begin{tabular}{llll}
\hline Semi-lattice & $L$ & set of sources & \\
Top & $\top$ & $\emptyset$ & \\
Initial value & init $(n)$ & $\emptyset$ & \\
Transfer function & $T F(n)$ & $\left\{\begin{array}{l}\text { add } \tau(n) \text { to set } \\
\text { identity }\end{array}\right.$ & if $n$ is a source \\
Meet operator & $\sqcap(x, y)$ & union $x \cup y$ & \\
Direction & & forward & \\
\hline
\end{tabular}

(a) Available source types.

\begin{tabular}{|c|c|c|c|}
\hline $\begin{array}{l}\text { Semi-lattice } \\
\text { Top } \\
\text { Initial value }\end{array}$ & $\begin{array}{l}L \\
\top \\
\operatorname{init}(n)\end{array}$ & $\begin{array}{l}\text { set of sinks } \\
\emptyset \\
\emptyset\end{array}$ & \\
\hline Transfer function & $T F(n)$ & $\left\{\begin{array}{l}\text { add } \tau(n) \text { to set } \\
\text { identity }\end{array}\right.$ & $\begin{array}{l}\text { if } n \text { is a sink } \\
\text { otherwise }\end{array}$ \\
\hline $\begin{array}{l}\text { Meet operator } \\
\text { Direction }\end{array}$ & $\sqcap(x, y)$ & $\begin{array}{l}\text { union } x \cup y \\
\text { backward }\end{array}$ & \\
\hline
\end{tabular}

(b) Anticipated sink types.

Figure 6. Available source types and anticipated sink types.

\begin{tabular}{lll}
\hline Semi-lattice & $L$ & bitvector of length $k$ \\
Top & $\top$ & $\overline{0}$ \\
Initial value & init $(n)$ & $\overline{0}$ \\
Transfer function & $T F(n)$ & $\left\{\begin{array}{l}\text { bit } i=1 \quad \text { if } n \text { is } S_{i} \text {-exclusive } \\
\text { identity otherwise }\end{array}\right.$ \\
$\begin{array}{ll}\text { Meet operator } \\
\text { Direction }\end{array}$ & $\sqcap(x, y)$ & $\begin{array}{l}\text { bitwise or } x \mid y \\
\text { backward }\end{array}$ \\
\hline
\end{tabular}

Figure 7. Computes exclusive_anticipated $(i)$.

$$
\begin{aligned}
& \text { for all } n \in N \text { do } \\
& \quad \text { sanitizers }=\operatorname{Project}(P, \text { available }(n) \text {, anticipated }(n)) \\
& \quad \text { for all } s \in \text { sanitizers do } \\
& \quad \operatorname{possible}(s)=\operatorname{possible}(s) \cup\{n\}
\end{aligned}
$$

The nodes that are $S_{i}$-exclusive are a subset of nodes that are $S_{i}$-possible. Computing $S_{i}$-exclusive nodes is a simple matter of removing from the set of $S_{i}$-possible nodes any node that is $S_{j}$-possible, for any $i \neq j$, as shown in Algorithm 2.

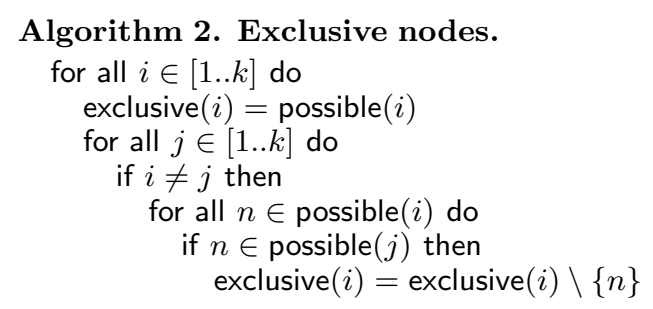

The last step is to compute latest-exclusive nodes. Recall that those are $S_{i}$-exclusive nodes that for some path from a source to a sink are the last $S_{i}$-exclusive node on that path. The idea for computing these nodes is to perform a backward dataflow analysis (Figure 7 ) that identifies, for each $S_{i}$, which nodes can reach an $S_{i}$-exclusive node. We write exclusive_anticipated $(i)$ for the set of nodes that can reach a $S_{i}$-exclusive node.

Latest-exclusive nodes are simply the set of exclusive nodes, minus the set of anticipated-exclusive nodes (Algorithm 3)

\section{Algorithm 3. Latest-exclusive nodes}

for all $i \in[1 . . k]$ do 


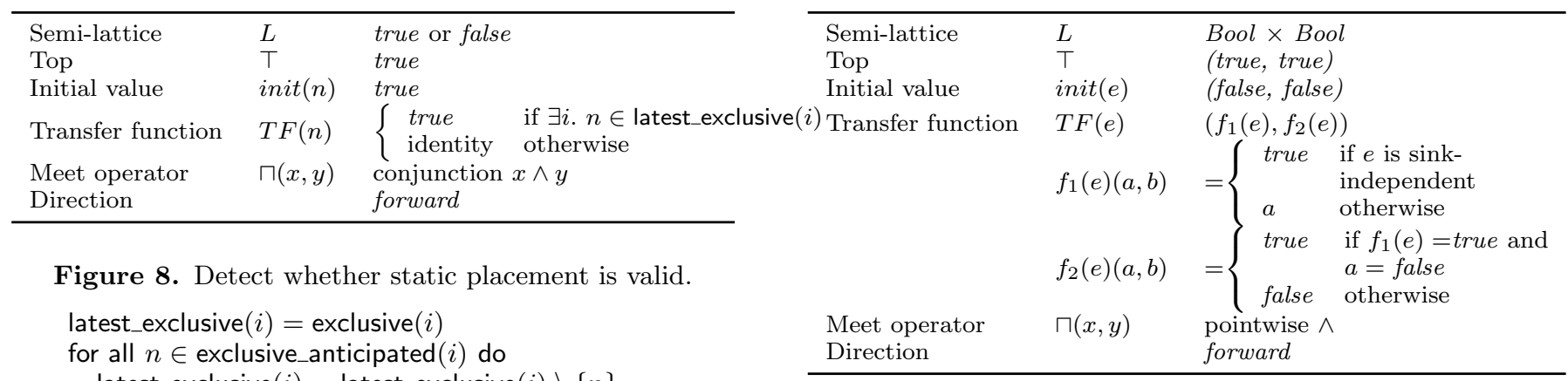

Figure 9. Computes sanitization $(e)$.

We place sanitizer $S_{i}$ at all nodes that are $S_{i}$-latestexclusive. Latest-exclusive nodes are generally somewhat rare, especially in dense graphs. For the graph in Figure 1, this algorithm will place sanitizers only at nodes $n_{1}, n_{13}$, and $n_{9}$. However, this is clearly insufficient, because not all values traversing the graph will be sanitized, such as values flowing from source node $n_{3}$ to sink node $n_{20}$.

It is simple to perform a dataflow analysis to detect whether all paths from sources to sinks go through a latestexclusive node. Figure 8 describes such an analysis. The dataflow facts are booleans, indicating whether all paths to the node have gone through a latest-exclusive node. (By construction, a path can have at most one latest-exclusive node, so there is no need to count the number of latestexclusive nodes on a path.) The static placement is valid if and only if the dataflow analysis produces a value of true at all sink nodes.

\subsection{Edge-based Placement}

In the rest of this section, we describe how to implement the edge-based solution, as advocated in Section 3.3. There are several different sets of edges that need to be identified, summarized in Figure 4. We present algorithms to compute each of these sets of edges.

Source- and sink-dependent edges: First, we compute the available source types and anticipated sink types for every edge, in a manner similar to the dataflow analyses in Figure 6. However, whereas Figure 6 is an analysis that computes dataflow facts for nodes, we need to compute dataflow facts for edges.

Next, for each edge $e$ we compute the edge policy $\mathcal{P}_{e}$ of the policy table $\mathcal{P}$ : it is policy table $\mathcal{P}$ restricted to the available source types and anticipated sink types of edge $e$. We use the edge projections to identify source-dependent and sink-dependent edges. Edge $e$ is source-dependent iff edge policy $\mathcal{P}_{e}$ has more than one unique sanitizer in any column. Edge $e$ is sink-dependent iff edge policy $\mathcal{P}_{e}$ has more than one unique sanitizer in any row.

In-trigger and out-trigger edges: Recall that in-trigger edges are source-independent edges with a source-dependent successor edge, and out-trigger edges are source-independent edges with a source-dependent predecessor edge. We can compute these edges efficiently purely by inspection of the dataflow graph. Let in trigger denote the set of in-trigger edges, and out_trigger denote the set of out-trigger edges.

Sanitization edges: Sanitization edges are sinkindependent edges that are the earliest sink-independent edge on some path from a source to a sink. They are the edges at which sanitization will be performed: at sink-independent edges the sanitization to apply to a value does not depend on which sink the value will go to.

Figure 10. Computes dom_sani( $e$ ): whether edge $e$ is dominated by sanitization edges.

Figure 9 presents a dataflow algorithm for computing sanitization edges. Note that the analysis computes dataflow facts for edges. Dataflow facts are pairs of boolean values. The first value is true for an edge if and only if all paths to the edge go through a sink-independent edge. The second boolean value is true for sanitization edges: edges that are the first sink-independent edge on some path, which is exactly the edges that are sink independent and have at least one path to it that doesn't go through a sink-independent edge. Note that the transfer function for edge $e$ is given as a pair of functions, $f_{1}(e)$ and $f_{2}(e)$, each of which is a function from the input dataflow fact (a pair of boolean values, $(a, b)$ ) to a boolean value, which forms part of the output dataflow fact.

Tag and untag edges: Tag and untag edges are where we, respectively, start and stop run-time tracking of values. Definitions of tag and untag edges rely on dominance, which is easily computed by flowing availability values forward, and using intersection as the meet operator.

The definition of both tag and untag edges relies on identifying edges that are dominated by a sanitization edge, for which we use the dataflow analysis in Figure 10. We write dom_sani(e) if edge $e$ is dominated by sanitization edges. Note that if $e$ is a sanitization edge, then dom_sani $(e)$ is true.

The following algorithm computes the set of tag and untag edges. Tag edges are in-trigger edges that are not dominated by sanitization edges. Untag edges are either sanitization edges, or out-trigger edges that are not dominated by sanitization edges.

\section{Algorithm 4. Tag and untag edges.}

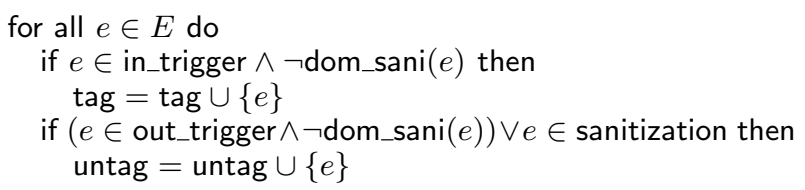




\begin{tabular}{|c|c|c|c|}
\hline Semi-lattice & $L$ & true or false & \\
\hline Top & $\top$ & false & \\
\hline Initial value & $\operatorname{init}(e)$ & false & \\
\hline Transfer function & $T F(e)$ & $\left\{\begin{array}{l}\text { true } \\
\text { false } \\
\text { identity }\end{array}\right.$ & $\begin{array}{l}\text { if } e \in \text { tag } \\
\text { if } e \in \text { untag } \\
\text { otherwise }\end{array}$ \\
\hline $\begin{array}{l}\text { Meet operator } \\
\text { Direction }\end{array}$ & $\sqcap(x, y)$ & $\begin{array}{l}x \vee y \\
\text { forward }\end{array}$ & \\
\hline
\end{tabular}

(a) Computes tag-available: reachable from tag edge without intervening untag edge.

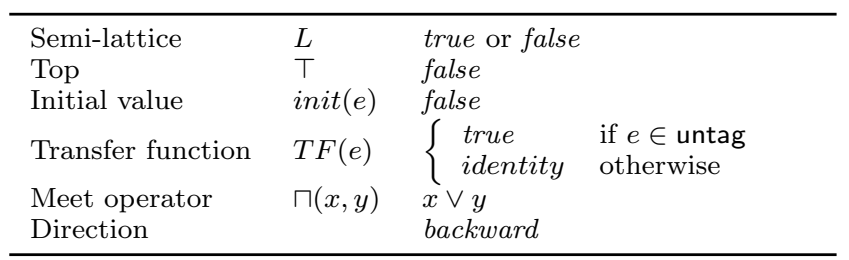

(b) Computes untag-anticipated: can reach untag edge.

Figure 11. Dataflow analyses for carry edge computation.

\begin{tabular}{|l|rrr|}
\hline Benchmark & DLLs & DLL (KB) & LOC \\
\hline \hline Alias Management & 3 & 65 & 10,812 \\
Chat Application & 3 & 543 & 6,783 \\
Bicycle Club App & 3 & 62 & 14,529 \\
Software & 15 & 118 & 11,941 \\
Sporting Field Management & 3 & 290 & 15,803 \\
Commitment Management & 7 & 369 & 25,602 \\
New Hire & 11 & 565 & 5,595 \\
Expense Report Approval & 4 & 421 & 78,914 \\
Customer Support Portal & 14 & 2,447 & 66,385 \\
Relationship Management & 5 & 3,345 & $1,810,585$ \\
\hline
\end{tabular}

Figure 12. Benchmark applications, sorted by code size.

Carry edges: Finally, carry edges are those on a path from a tag to an untag edge that does not pass through an untag edge. The set of carry edges cab be computed by first performing a forward dataflow analysis to compute tagavailable - the set of edges that are reachable from a tag edge without an intervening untag edge - and then performing a backward dataflow analysis to compute untag-anticipated, the set of edges that can reach an untag edge. These dataflow analyses are shown in Figure 11. Carry edges are the nontag, non-untag edges that are in both the tag-available and untag-anticipated sets, as defined in the following algorithm.

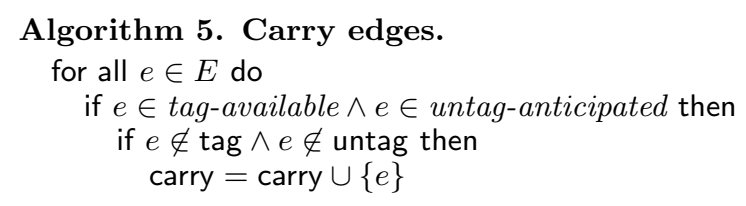

\section{Experimental Evaluation}

Our experimental evaluation focuses on showing how our algorithms achieve automatic sanitizer placement and reduce the number of instrumentation points needed compared to a baseline implementation that performs dynamic taint tracking between all sources and sinks. Our target class of applications is long-running server applications. Runtime overhead of taint tracking is generally very workload-specific (e.g., [9]), so we choose not to evaluate runtime overhead directly. Reducing the number of instrumentation points is a valuable goal, because long-running applications with diverse workloads will have very high code coverage over time, hitting increasingly many instrumentation points. Section 5.1 presents the results of applying our techniques to large C\# web applications written in ASP.NET. Section 5.2 provides results of applying our approach to large, synthetically constructed graphs.

\section{$5.1 \quad$ Large Applications}

Figure 12 contains a summary of information about our macro-benchmarks. These are relatively large business web applications written on top of the ASP.NET framework, consisting of several separate DLLs, as shown in column 2. Not all code contained within the application source tree is actually deployed to the Web server. Most of the time, the number and size of deployed DLLs primarily consisting of .NET bytecode is a good measure of the application size, as shown in column 3. Note that in a several cases, libraries supplied in the form of DLLs without the source code constitute the biggest part of an application. Finally, to provide another measure of the application size, column 4 shows the traditional line-of-code metric for all the code within the application.

Policy: There are applications ranging from tens of thousands of lines of code to over a million in the case of the Relationship Management application. We classified sources and sinks into the three categories: normal, file, resource, based on their functionality (i.e. TextWriter.Write is a file-related sink). We used the policy shown to the right for these applications. Finally, we completely disregarded existing sanitizers, fully automating sanitizer placement.

\subsubsection{Node-based Placement}

Figure 13 contains the results of applying node-based analysis described in Figure 3.2. Applications are represented as graphs, some nodes of which are marked as sources or sinks. We are operating on a fairly sparse graph representation of the program computed by the CAT.NET tool [22]. Nodes of the graph are parameters and return results of individual methods in the application or its libraries. Edges represent data flow as inferred by CAT.NET. A different static analysis tool could also be used to construct these graphs; the precision and soundness of CAT.NET results is orthogonal to our approach. The number of nodes (column 2) as well as sources and sinks (3-4) ranges from dozens to lower thousands.

Columns 5-8 summarize information about tainted nodes in the graph. Column 5 is forward-tainted nodes (available). Column 6 is backward-tainted nodes (anticipated). Column 7 is both forward- and backward-tainted nodes and column 8 is the fraction of these nodes compared to all nodes in the graph. We can see that for a well-connected graph, the percentage of such nodes can be quite high, going higher than $60 \%$. The implication is that a very high fraction of nodes needs to be instrumented to propagate the taint forward at runtime.

Columns 9-11 capture our exclusive node computation. Column 9 is the number of exclusive nodes and column 10 is the number of latest exclusive nodes. Column 11 is the fraction of latest exclusive nodes within the nodes of the graph. Finally, column 12 shows the coverage, which is the fraction of all source-sink paths that are properly sanitized 


\begin{tabular}{|c|c|c|c|c|c|c|c|c|c|c|c|}
\hline \multirow[b]{2}{*}{ Application } & \multirow{2}{*}{$\begin{array}{r}\text { Graph } \\
\text { nodes }\end{array}$} & \multicolumn{2}{|c|}{ Taint } & \multicolumn{4}{|c|}{ Tainted nodes } & \multicolumn{3}{|c|}{ Exclusive nodes } & \multirow{2}{*}{$\begin{array}{c}\text { Sanitization } \\
\text { coverage }\end{array}$} \\
\hline & & sources & sinks & forward & backward & both & ratio & all & l.e. & ratio & \\
\hline Terralever & 156 & 64 & 69 & 140 & 140 & 76 & $48 \%$ & 122 & 50 & $32 \%$ & $82 \%$ \\
\hline Alias Management & 59 & 11 & 12 & 22 & 21 & 11 & $18 \%$ & 19 & 9 & $15 \%$ & $86 \%$ \\
\hline Contoso Bicycle Club & 161 & 50 & 54 & 145 & 133 & 87 & $54 \%$ & 94 & 40 & $24 \%$ & $50 \%$ \\
\hline Windows Experience Catalog & 204 & 47 & 83 & 186 & 160 & 101 & $49 \%$ & 120 & 49 & $24 \%$ & $93 \%$ \\
\hline Commitment Management & 356 & 135 & 132 & 299 & 296 & 183 & $51 \%$ & 221 & 86 & $24 \%$ & $79 \%$ \\
\hline New Hire & 502 & 142 & 183 & 401 & 409 & 229 & $45 \%$ & 275 & 110 & $21 \%$ & $70 \%$ \\
\hline Expense Report Approval & 805 & 214 & 322 & 722 & 637 & 408 & $50 \%$ & 389 & 170 & $21 \%$ & $82 \%$ \\
\hline Customer Support Portal & 3,881 & 967 & 1,219 & 3,488 & 3,263 & 2,266 & $58 \%$ & 1,721 & 770 & $19 \%$ & - \\
\hline Relationship Management & 3,639 & 1,054 & 982 & 3,321 & 3,104 & 2,241 & $61 \%$ & 1,565 & 637 & $17 \%$ & - \\
\hline
\end{tabular}

Figure 13. Node-based analysis and its effectiveness.

\begin{tabular}{|c|c|c|c|c|c|c|c|c|c|c|c|c|c|c|c|c|c|}
\hline Application & 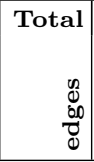 & $\begin{array}{c}\text { Tai } \\
0 \\
\bigcup_{0}^{0} \\
\vdots \\
0 \\
0\end{array}$ & int & 3 & Taint & مأ & $\underset{\sim}{\stackrel{0}{*}}$ & 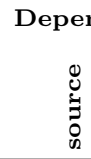 & adent & $\Xi$ & rigger & 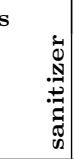 & \begin{tabular}{l}
80 \\
\multirow{+}{*}{}
\end{tabular} & 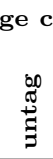 & count & Ins & \\
\hline Terralever & 142 & 96 & 89 & 140 & 137 & 136 & $95 \%$ & 3 & 1 & 8 & 0 & 97 & 1 & 5 & 0 & 6 & 22.66 \\
\hline Alias Management & 962 & 11 & 10 & 13 & 14 & 13 & $1 \%$ & 0 & 0 & 0 & 0 & 11 & 0 & 2 & 0 & 2 & 6.5 \\
\hline Contoso Bicycle Club & 182 & 80 & 70 & 170 & 174 & 164 & $90 \%$ & 32 & 27 & 29 & 9 & 84 & 2 & 6 & 7 & 15 & 10.93 \\
\hline Windows Experience & 430 & 68 & 162 & 386 & 364 & 358 & $83 \%$ & 133 & 125 & 14 & 117 & 193 & 2 & 31 & 123 & 156 & 2.29 \\
\hline Commitment Management & 461 & 177 & 188 & 420 & 409 & 386 & $83 \%$ & 110 & 114 & 45 & 41 & 215 & 6 & 30 & 108 & 144 & 2.68 \\
\hline New Hire & 873 & 258 & 347 & 680 & 771 & 652 & $74 \%$ & 181 & 192 & 108 & 75 & 342 & 27 & 67 & 126 & 220 & 2.96 \\
\hline Expense Report Approval & 1,389 & 367 & 503 & 1,286 & 1,296 & 1,208 & $86 \%$ & 189 & 197 & 131 & 143 & 577 & 23 & 99 & 144 & 266 & 4.54 \\
\hline Customer Support Portal & 8,985 & 1,505 & 2,167 & 8,534 & 8,469 & 8,069 & $89 \%$ & 4,315 & 4,364 & 901 & 1,331 & 2,875 & 232 & 541 & 3,917 & 4,690 & 1.72 \\
\hline Relationship Management & 17,732 & 2,376 & 2,594 & 17,227 & 17,358 & 16,888 & $95 \%$ & 11,227 & 11,585 & 2,057 & 2,020 & 4,407 & 428 & 533 & 10,725 & 11,686 & 1.44 \\
\hline
\end{tabular}

Figure 14. Edge-based analysis and its effectiveness. Reduction in number of instrumented edges is shown in last column.

with latest exclusive nodes. ${ }^{2}$ Two key take-aways from this table are as follows:

- node-based instrumentation is very expensive, with as many as $60+\%$ of nodes needing to be instrumented; and

- the obtained coverage is significantly less than $100 \%$, the static node-based approach is generally unacceptable for sanitizer placement.

\subsubsection{Edge-based Placement}

Figure 14 shows the results of edge-based analysis. Column 2 shows the number of edges in the graph. Columns 3-4 show the number of sources and sinks, respectively. Columns 57 show the number of forward and backward-tainted edges and edges tainted in both directions. Column 8 shows the percentage of edges tainted in both directions as a fraction of the number of edges in the graph.

Columns 9-10 show the number of source- and sinkdependent edges. Columns 11-13 show the number of inand reverse trigger edges and sanitizer edges. Columns 14-16 show the counts for the other kinds of edges computed by the edge-based formulation. Finally, columns 17 and 18 show the number of edges needing instrumentation and the savings compared to the naïve approach of instrumenting edges that are both forward- and backward-tainted. (As described in Section 3.3.5, we do not count unneeded sanitization edges when counting edges that require instrumentation.) Two key take-aways from this table are as follows:

- We see that for most applications, the percentage of edges that are forward- and backward-tainted is quite high, indicating that the underlying dataflow analysis of CAT.NET is quite imprecise, leading to a great deal of connectivity within the dataflow graph.

\footnotetext{
${ }^{2}$ Coverage numbers are not available for the largest two application; the large number of paths in the dataflow graph cannot be enumerated in reasonable time.
}

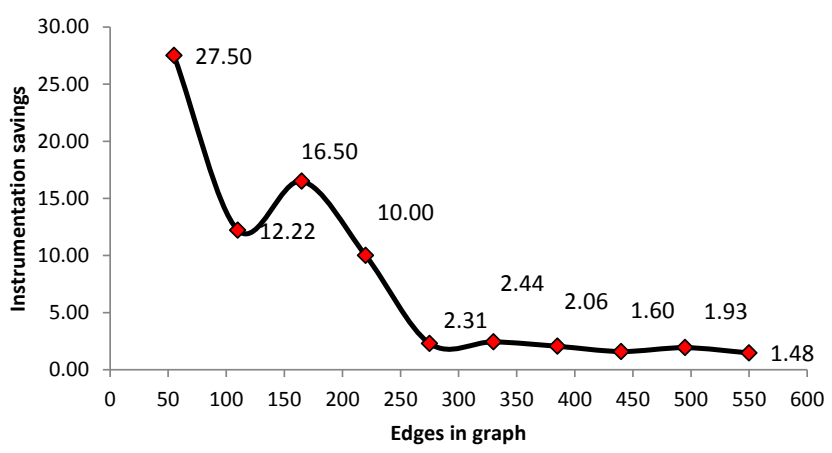

Figure 15. Synthetic graph results.

- In general, our analysis is not as effective at reducing the number of instrumentation points for densely-connected graphs (the last several rows) as it is for the sparser graphs (the first several rows).

\subsection{Synthetic Graphs}

Finally, we evaluate our (edge-based) algorithm on some synthetically constructed graphs. To build such graphs, we start with 100 sources, 100 sinks, and 1,000 regular nodes. We randomize the type of the sources with equal probability between $\oslash, \square, \bigcirc, \diamond$, and $\triangle$, and the type of sinks between $\oslash, \boldsymbol{\square}, \mathbf{\square}$, and $\boldsymbol{\Lambda}$, using the policy in Figure 2 for proper sanitizer placement. We connect sources to sinks by performing a random walk of length 10 starting at a random source and ending at a random sink through the graph, creating edges as we pass from node to node. We use a density parameter $d$ to vary how many such walks we perform, affecting the number of edges.

Figure 15 shows the improvements with edge-based instrumentation compared to naïve, taint-based instrumentation as the number of edges grows. We can see that for sparse graphs, the improvements are most noticeable, peaking at over $27 \times$, gradually becoming less pronounced (only $48 \%$ im- 
provement for 550 edges). This suggests that sparser graphs constructed with the help of a precise analysis are likely to benefit the most, whereas graphs that are overly connected as a result of analysis imprecision benefit less. This observation further justifies investement in precise static tainting.

\section{Related Work}

Because of space limitations, we only provide a brief summary of related work.

Software security analysis of web applications: Program analysis has a long history of being used for finding security bugs in web applications. Static analysis has been advocated for PHP, Java, and other languages [18, 40, 14, 13]. Multiple runtime analysis systems for information flow tracking have also been proposed [21, 10, 25, 27].

Automating placement: Most recently, we have seen increased interest in automating security-critical decisions for the developer $[32,39]$. The use of security type system for enforcing correctness is another case of cooperating with the developer to achieve better code quality and correctness guarantees [29].

Sanitizer correctness: Balzarotti et al. show that custom sanitizer routines are often incorrectly implemented [3]. Our concerns in this paper are complimentary to sanitizer correctness. The Cross-Site Scripting Cheat Sheet shows over two hundred examples of strings that exercise common corner cases of web sanitizers [30]. The BEK project proposes a systematic domain-specific languages for writing and checking sanitizers [11, 38].

Specification inference: Livshits et al. propose an approach to inferring information flow specifications (sources, sanitizers, and sinks) using factor graphs [19]. Kremenek et al. [16] propose belief inference as a way to infer specifications for static analysis checkers. Vaughan et al. propose policy inference to discover correct declassification policies [37].

Graph algorithms: LCM and PRE: A range of graphtheoretical algorithms from compiler literature is relevant for our work. In particular, Knoop et al. [15] describe lazy code motion. Rüthing et al. describe a variant of it called sparse code motion [31]. Partial redundancy elimination of PRE is described in Hosking et al. $[12,5]$.

\section{Conclusions}

Despite years of research in explicit information flow (or tainting), practical deployment has been scarce. One of the obstacles is the overhead that taint tracking induces at runtime. In this paper, we propose a set of algorithms that drastically reduce the need to track taint throughout the application. Our algorithms are graph-theoretical, run fast, and readily integrate with other approaches to tracking explicit information flow.

We discover that while node-based taint tracking is easier to implement, it both requires too many nodes to be instrumented and does not generally provide full sanitization coverage. Edge-based placement is an alternative that provides full sanitization, resulting in a reduction of nodes needing instrumentation, sometimes as significant as $27 \times$, compared to the naïve, tainting-based strategy.

\section{References}

[1] A. V. Aho, M. Lam, R. Sethi, and J. D. Ullman. Compilers: Principles, Techniques, and Tools. Addison-Wesley, 2007.
[2] D. Avots, M. Dalton, B. Livshits, and M. S. Lam. Improving software security with a $\mathrm{C}$ pointer analysis. In Proceedings of the 27th International Conference on Software Engineering (ICSE), pages 332-341. ACM, May 2005.

[3] D. Balzarotti, M. Cova, V. Felmetsger, N. Jovanovic, E. Kirda, C. Kruegel, and G. Vigna. Saner: Composing Static and Dynamic Analysis to Validate Sanitization in Web Applications. In Proceedings of the IEEE Symposium on Security and Privacy, Oakland, CA, May 2008.

[4] D. Bates, A. Barth, and C. Jackson. Regular expressions considered harmful in client-side XSS filters. International World Wide Web Conference ( $W W W$ ), 2010.

[5] P. Briggs and K. D. Cooper. Effective partial redundancy elimination. In Proceedings of the ACM SIGPLAN 1994 conference on Programming language design and implementation, PLDI '94, pages 159-170, 1994. ISBN 0-89791-662-X.

[6] B. Chess and J. West. Dynamic taint propagation: Finding vulnerabilities without attacking. Information Security Technical Reports, 13:33-39, January 2008. ISSN 1363-4127.

[7] E. Chin and D. Wagner. Efficient character-level taint tracking for java. In Proceedings of the 2009 ACM workshop on Secure web services, SWS '09, pages 3-12, 2009.

[8] S. Chong, K. Vikram, and A. C. Myers. Sif: enforcing confidentiality and integrity in Web applications. In Proceedings of USENIX Security Symposium, 2007.

[9] W. Enck, P. Gilbert, B.-G. Chun, L. P. Cox, J. Jung, P. McDaniel, and A. N. Sheth. Taintdroid: an information-flow tracking system for realtime privacy monitoring on smartphones. In Proceedings of the USENIX Conference on Operating Systems Design and Implementation, pages 1-6, 2010.

[10] V. Haldar, D. Chandra, and M. Franz. Dynamic taint propagation for Java. In Proceedings of the Annual Computer Security Applications Conference, Dec. 2005. ISBN 0-7695-2461-3. doi: http://dx.doi.org/10.1109/CSAC.2005.21.

[11] P. Hooimeijer, B. Livshits, D. Molnar, P. Saxena, and M. Veanes. Fast and precise sanitizer analysis with BEK. In Proceedings of the Usenix Security Symposium, Aug. 2011.

[12] A. L. Hosking, N. Nystrom, D. Whitlock, Q. Cutts, and A. Diwan. Partial redundancy elimination for access path expressions. Software Practice and Experience, 31:577-600, May 2001.

[13] Y.-W. Huang, F. Yu, C. Hang, C.-H. Tsai, D.-T. Lee, and S.-Y. Kuo. Securing Web application code by static analysis and runtime protection. In Proceedings of the International Conference on World Wide Web, 2004. ISBN 1-58113-844-X.

[14] N. Jovanovic, C. Kruegel, and E. Kirda. Pixy: A static analysis tool for detecting Web application vulnerabilities (short paper). In Proceedings of the IEEE Symposium on Security and Privacy, 2006. ISBN 0-7695-2574-1. doi: http://dx.doi.org/10.1109/ SP.2006.29.

[15] J. Knoop, O. Rüthing, and B. Steffen. Lazy code motion. SIGPLAN Not., 39:460-472, April 2004.

[16] T. Kremenek, P. Twohey, G. Back, A. Y. Ng, and D. R. Engler. From uncertainty to belief: Inferring the specification within. In Symposium on Operating Systems Design and Implementation, pages 161-176, Nov. 2006.

[17] B. Livshits and S. Chong. Towards fully automatic placement of security sanitizers and declassifiers. Technical report, Harvard University, 2012.

[18] B. Livshits and M. S. Lam. Finding security errors in Java programs with static analysis. In Proceedings of the Usenix Security Symposium, 2005.

[19] B. Livshits, A. V. Nori, S. K. Rajamani, and A. Banerjee. Merlin: Specification inference for explicit information flow problems. In Proceedings of the Conference on Programming Language Design and Implementation, June 2009.

[20] M. Martin, B. Livshits, and M. S. Lam. Finding application errors and security flaws using PQL: a program query language. In Proceedings of the Conference on Object Oriented Programming Systems Languages and Applications, pages 365-383, 2005. ISBN 1-59593-031-0.

[21] M. Martin, B. Livshits, and M. S. Lam. SecuriFly: runtime vulnerability protection for Web applications. Technical report, Stanford University, 2006.

[22] Microsoft Corporation. Microsoft Code Analysis Tool .NET (CAT.NET). http://www.microsoft.com/en-us/download/ details . aspx?id=19968, 32009. 
[23] Microsoft Corporation. Microsoft web protection library. http: //wpl.codeplex.com/, 2012.

[24] N. Mitchell, G. Sevitsky, and H. Srinivasan. The diary of a datum: an approach to modeling runtime complexity in frameworkbased applications. In Proceedings of the European Conference on Object-Oriented Programming, Systems, Languages, and Applications, 2005.

[25] A. Nguyen-Tuong, S. Guarnieri, D. Greene, J. Shirley, and D. Evans. Automatically hardening Web applications using precise tainting. In Proceedings of the IFIP International Information Security Conference, 2005.

[26] OWASP. OWASP-Java-HTML-sanitizer. http://code.google. com/p/owasp-java-html-sanitizer/, 2011.

[27] T. Pietraszek and C. V. Berghe. Defending against injection attacks through context-sensitive string evaluation. In Proceedings of the Recent Advances in Intrusion Detection, Sept. 2005.

[28] W. Robertson and G. Vigna. Static enforcement of web application integrity through strong typing. In Proceedings of the 18 th conference on USENIX security symposium, pages 283298, 2009.

[29] W. Robertson and G. Vigna. Static enforcement of web application integrity through strong typing. In Proceedings of the Usenix Security Symposium, Aug. 2009.

[30] RSnake. XSS cheat sheet for filter evasion. http://ha.ckers.org/ xss.html.

[31] O. Rüthing, J. Knoop, and B. Steffen. Sparse code motion. In Proceedings of the 27th ACM SIGPLAN-SIGACT symposium on Principles of programming languages, POPL '00, pages 170 183, 2000.

[32] M. Samuel, P. Saxena, and D. Song. Context-sensitive autosanitization in web templating languages using type qualifiers. In Proceedings of the Conference on Computer and Communications Security, Oct. 2011.

[33] P. Saxena, D. Molnar, and B. Livshits. ScriptGard: Automatic context-sensitive sanitization for large-scale legacy web applications. In Proceedings of the Conference on Computer and Communications Security, Oct. 2011.

[34] V. Srivastava, M. D. Bond, K. S. McKinley, and V. Shmatikov. A security policy oracle: detecting security holes using multiple API implementations. In Proceedings of the 32nd ACM SIGPLAN conference on Programming language design and implementation, PLDI '11, pages 343-354, 2011. ISBN 978-1-4503-0663-8.

[35] Z. Su and G. Wassermann. The essence of command injection attacks in Web applications. In Proceedings of the Symposium on Principles of Programming Languages, 2006. ISBN 1-59593027-2.

[36] O. Tripp, M. Pistoia, S. J. Fink, M. Sridharan, and O. Weisman. TAJ: effective taint analysis of web applications. In Proceedings of the Conference on Programming Language Design and Implementation, pages 87-97, 2009.

[37] J. Vaughan and S. Chong. Inference of expressive declassification policies. In Proceedings of IEEE Symposium on Security and Privacy, pages $180-195$, May 2011.

[38] M. Veanes, P. Hooimeijer, B. Livshits, D. Molnar, and N. Bjorner. Symbolic finite state transducers: Algorithms and applications. In Proceedings of the Sympolisium on Principles of Programming Languages (POPL), Jan. 2012.

[39] J. Weinberger, P. Saxena, D. Akhawe, M. Finifter, R. Shin, and D. Song. A systematic analysis of XSS sanitization in web application frameworks. In Proceedings of the European Symposium on Research in Computer Security, Sept. 2011.

[40] Y. Xie and A. Aiken. Static detection of security vulnerabilities in scripting languages. In Proceedings of the Usenix Security Symposium, 2006.

[41] E. Z. Yang. Html purifier. http://code.google.com/p/ owasp-java-html-sanitizer/, 2011. 